\title{
CARBON STARS AS PLANETARY NEBULA PROGENITORS
}

\author{
G. R. Knapp \\ Department of Astrophysical Sciences \\ Princeton University \\ Princeton, NJ 08544, U.S.A.
}

\begin{abstract}
Molecular line observations show that some planetary nebulae are still only partially ionized and are surrounded by the remains of the mass loss envelope shed by the preceding AGB star. The mass loss rates and outflow velocities of these envelopes are similar to those of the cool winds from luminous AGB stars. Both the kinematics of carbon stars and observations of the molecular envelopes around young planetaries show that the carbon star progenitors have a wide range of ages and of mass loss rates. There is increasing evidence that a significant fraction of AGB stars are carbon stars and that these provide a substantial contribution to the total mass returned to the interstellar medium.
\end{abstract}

\section{INTRODUCTION}

- The advent of infrared and millimeter-wavelength astronomy in the past twenty years has allowed the study of the cool, dusty molecular winds shed by asymptotic giant branch stars. These observations yield a wealth of information relating to many aspects of galactic and stellar evolution and of the chemistry of the interstellar medium. Such topics as the rate of mass return, the chemical composition of the returned mass, and the formation of dust are under intensive study.

Mass loss from an AGB star takes place, to zeroth order, as a spherically symmetric, steady wind whose outflow velocity becomes constant at distances of a few stellar radii. Observed values of the wind velocities range from 3 to $80 \mathrm{~km} / \mathrm{sec}$, but most objects observed so far have winds with velocities of $10-25 \mathrm{~km} / \mathrm{sec}$. The mass loss rates likewise have a large range, a few $\times 10^{-8} \mathrm{M}_{\odot} \mathrm{yr}^{-1}$ to $>10^{-4} \mathrm{M}_{0} \mathrm{yr}^{-1}$, but "typical" values are in the few $\times 10^{-6}$ to $10^{-5} \mathrm{M}_{\Theta} \mathrm{yr}^{-1}$ range.

These winds are observed in their infrared excess due to dust, the silicate and SiC absorption or emission features, and via a large number of molecular lines. Of these, the most useful for studies of mass loss rates etc. are the $C 0$ rotational millimeter and sub-millimeter lines, which are observed from both carbon stars and $M$ stars (stars with $n(0)>n(C)$, hereafter "oxygen" stars), and the 1612 
$\mathrm{MHz}$ OH maser line, observed from oxygen stars only. There can now be little doubt that it is this mass shed by the AGB star which is destined to become a planetary nebula as it becomes ionized by the hot degenerate core at the end of the mass loss phase; several planetary nebulae still have the remains of the circumstellar molecular envelope, as shown by observations of the $\mathrm{CO}$ lines, $\mathrm{H}_{2}$ emission, $\mathrm{OH}$ maser emission, and dust.

The resulting mass-loss envelopes can reach considerable extents, $>1$ pc in radius, before being physically truncated by the interstellar pressure or chemically truncated by ultraviolet radiation. The radial structure of the objects thus contains information about their time evolution, which is now being exploited as high-resolution instruments come on line. This review will first summarize the observational data for carbon stars. The local galactic kinematics and distribution will then be compared with those of planetary nebulae. The distribution of. the chemical composition of planetary nebulae will be compared with that of AGB stars, and finally observations of planetary nebulae which still contain molecular material will be discussed and an evolutionary sequence constructed.

\section{I . OBSERVATIONAL DATA - GLOBAL CO PROFILES}

From the point of view of the appearance of the co profiles, oxygen stars, S stars, carbon stars and planetary nebulae are identical. Figure 1 shows the $\mathrm{CO}(1-0)$ line profiles for IRC+00509 (oxygen star), CIT6 (carbon star), RY Dra (carbon star) and NGC7027 (planetary nebula), measured with the AT\&T Bell Labs. 7m telescope. These profiles give the central velocity and velocity width of the outflow, and the intensity also gives the mass loss rate. A few years ago, observational sensitivity limits meant that only the stars which had the highest mass loss rates (or those few which are very nearby, $<200$ pc) were detected in the CO line, and the sample of AGB stars for which these data were available was unrepresentative. This is still somewhat true, but is rapidly improving. The very successful IRAS satellite mission (Neugebauer et al. 1984) provided a very large sample of target evolved stars (01non et al. 1984) which are being intensively observed in the $\mathrm{CO}$ and $\mathrm{OH}$ lines (Zuckerman and Dyck 1986a,b; Habing and te Lintel Hekkert 1987; Eder et al. 1987). In addition, observational sensivity has greatly improved for $\mathrm{CO}$ observations, making accessible measurements of a wider range of mass loss rates (e.g. 0lofsson et al. 1987). At the present time, Co emission has been detected from over two hundred evolved stars, including nearly twenty planetary- and proto-planetary nebulae, and eventually it is likely that detections of several hundred stars will be made.

\section{THE LOCAL KINEMATICS OF CARBON STARS}

The huge data base on the mid-and far-infrared emission from carbon stars provided by the IRAS satellite (IRAS Point Source Catalog 1985) has led to the study the galactic distribution of evolved stars. Recent analyses have been undertaken by Thronson et al. (1987), 

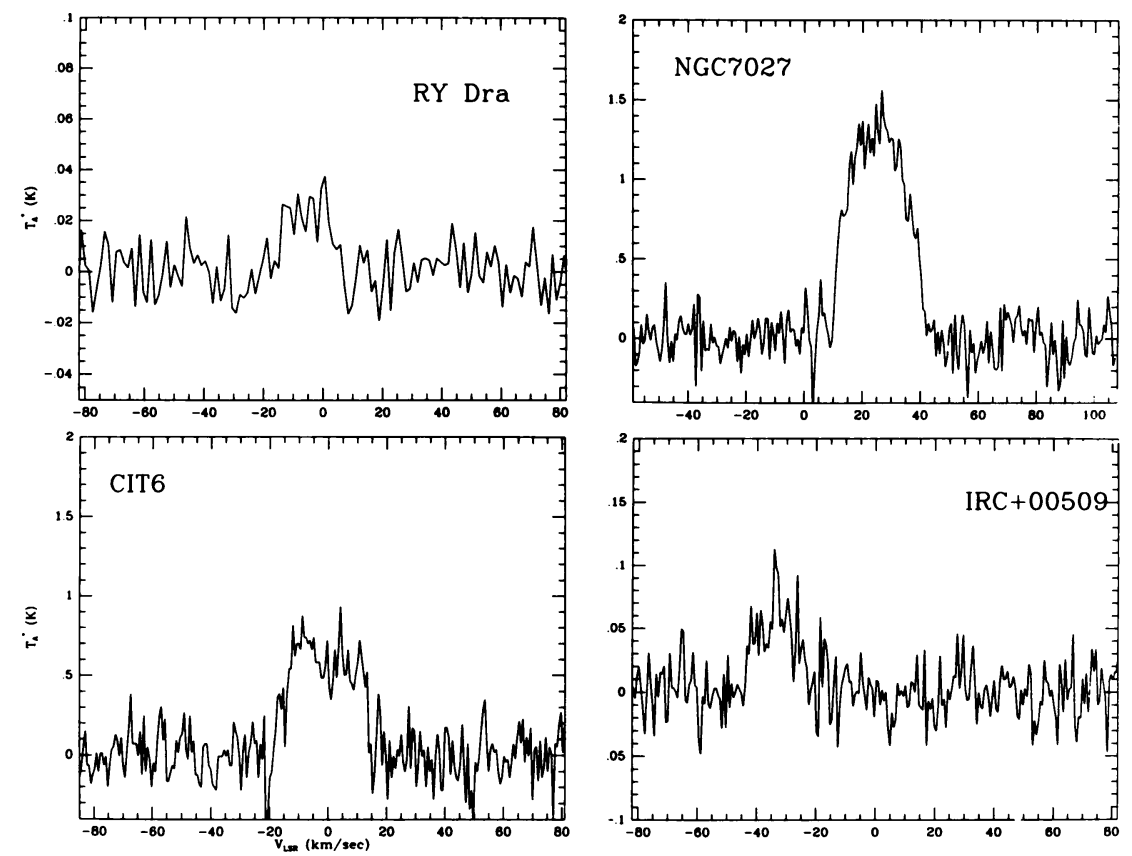

Figure 1. $C O(1-0)$ line profiles of RY Dra (carbon star), NGC7027 (planetary nebula), CIT6 (carbon star) and IRC+00509 (oxygen star) measured with the AT\&T Bell Laboratories $7 \mathrm{~m}$ telescope.

Claussen et al. (1987) and Willems and de Jong (1987). What is needed at present is a measure of the absolute luminosity (or luminosity function) of carbon stars. The present discussion concentrates on the local distribution, i.e. the scale height and velocity dispersion of the local carbon stars.

Figure 2 shows the radial velocity histogram for carbon stars, where the radial velocities are measured using the $\mathrm{CO}(1-0)$ or $(2-1)$ lines. The present set of observations has fairly good sky coverage. While objects in the northern hemisphere have been more extensively observed, the coming on line of the submillimeter telescopes on Hawaii has enabled searches for CO emission to be extended as far south as $\delta=$ $-60^{\circ}$ (Phillips et al. 1987). The data in Figure 2 are taken from 


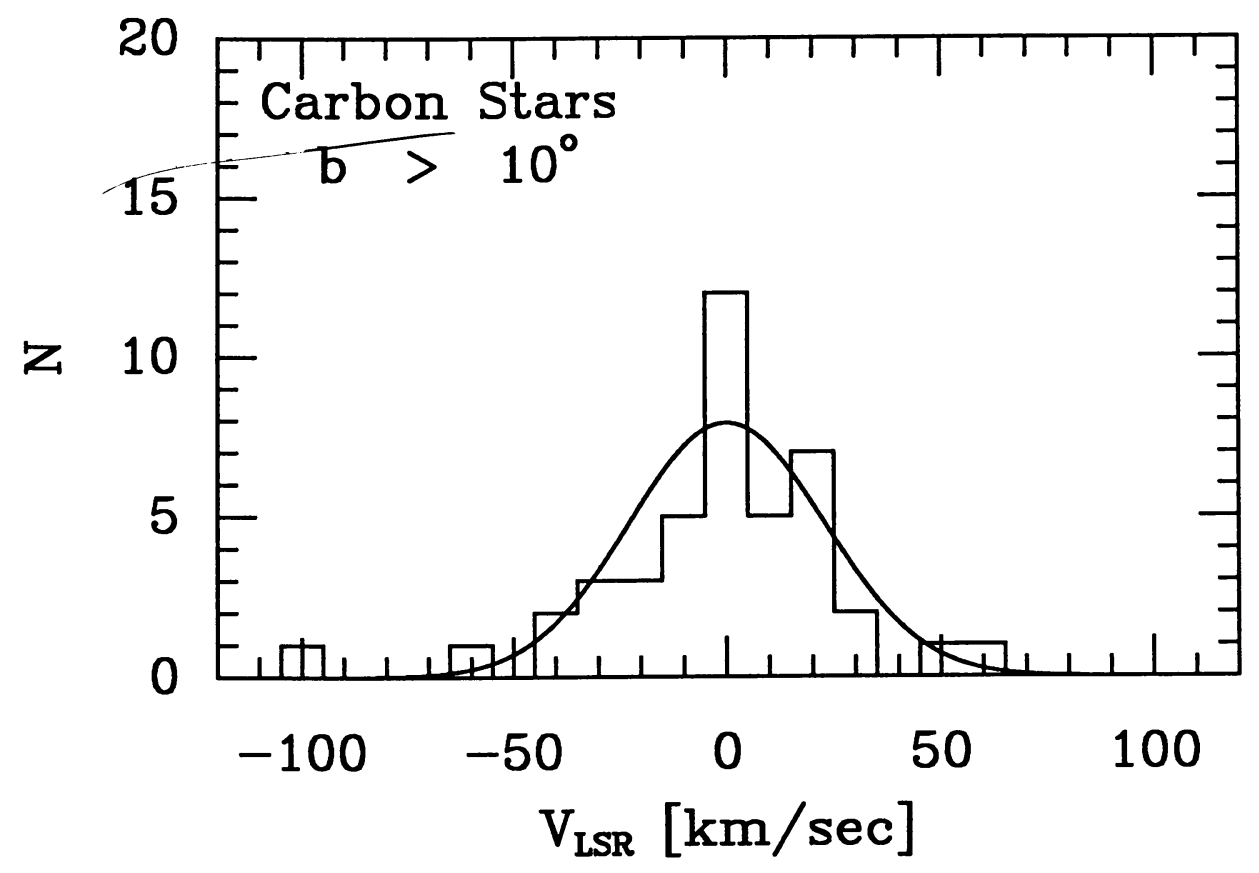

Figure 2. Histogran of radial velocities with respect to the Local Standard of Rest for carbon stars with $|\mathrm{b}|>10^{\circ}$. The radial velocities are measured using emission in the $\mathrm{CO} \mathrm{J}=$ 1-0 and 2-1 1ines.

papers by Arquilla et al. (1987), Leahy et al. (1987), Rieu et al. (1987), Huggins and Healy $(1986 a, b)$, Knapp and Morris (1985), Knapp (1986, and unpublished), 0lofsson et al. (1987), Phillips et al., (1987), Wannier and Sahai (1987), Zuckerman and Dyck (1986a,b, 1987) and Zuckerman, Dyck and Claussen (1986), and total 209 objects. For Figure 2, only stars with $|\mathrm{b}|>10^{\circ}$ are plotted, to avoid galactic rotation effects. Assuming that the velocities are isotropic, $\checkmark(3$-dimensional $)=39.3+5.9 \mathrm{~km} / \mathrm{sec}$ for the carbon stars. Figure 2 also shows this gaussian plotted on top of the histogram. It is a reasonable fit, though the data marginally suggest the presence of components of different velocity dispersion. A slightly higher value, $v=49.5 \pm 6.7 \mathrm{~km} / \mathrm{sec}$, is found for the oxygen stars, suggesting that the mass-losing carbon stars are, as a group, marginally younger than the oxygen stars.

Zuckerman and Dyck (1986b) have pointed out that carbon stars with large outflow velocities tend to be found close to the galactic plane, implying that they have a small scale height and therefore arise from a 


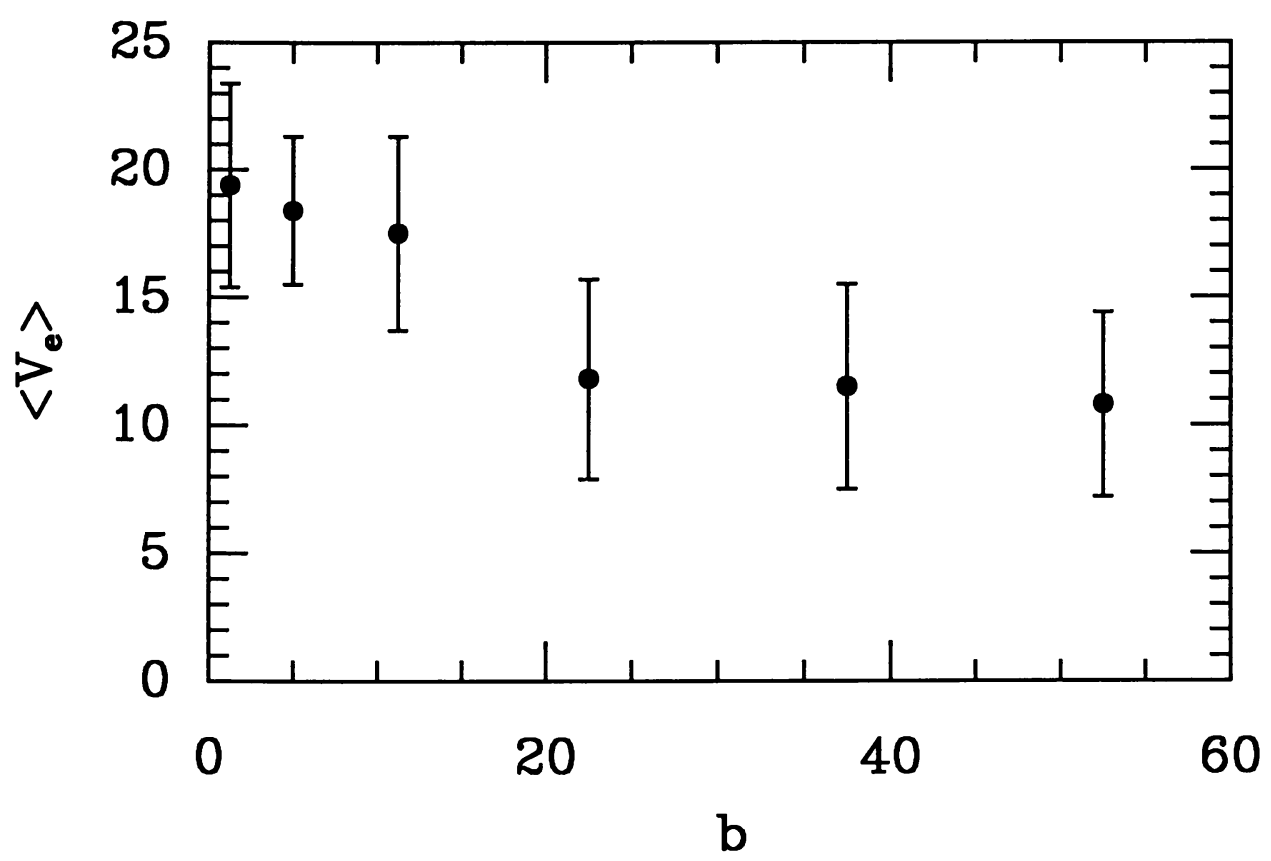

Figure 3. Mean expansion velocity for winds from carbon stars, binned by the galactic latitude of the stars.

population of larger progenitor mass. This trend is illustrated in Figure 3, where the mean carbon star outflow velocity is plotted versus latitude. These data suggest that carbon stars, like oxygen stars, have a range of progenitor masses and hence a range of ages.

The three-dimensional velocity distribution of local carbon stars has a dispersion of $\sim 40 \mathrm{~km} / \mathrm{sec}$. From the compilation of Mihalas and Binney (1981) the progenitor mass of the carbon star is likely to be $1.5 \mathrm{M}_{\Theta}$, in agreement with the conclusions of Claussen et al (1987). This velocity dispersion is significantly less than that of opticallyobserved carbon stars ( $56 \mathrm{~km} / \mathrm{sec}$, Mihalas and Binney 1981) or of planetary nebulae and white dwarfs $(\sim 60 \mathrm{~km} / \mathrm{sec})$. This is further evidence that carbon stars arise from a range of progenitor masses; those losing mass copiously enough to detect in the $\mathrm{CO}$ line have higher progenitor masses than the average carbon star.

IV. THE FRACTION OF AGB STARS WHICH ARE CARBON STARS

Zuckerman and Aller (1986) have shown that, of the planetary nebulae with reasonably well-measured abundances, more than $50 \%$ have $n(C)>$ 
$\mathrm{n}(0)$. There is now gruwing evidence that many (most?) AGB stars may evolve from "oxygen" to carbon stars. (1) Willems and de Jong (1986) and Little-Marenin (1986) have shown that some J-type carbon stars have silicate dust in their envelopes, suggesting that the stellar atmosphere has just changed composition from oxygen to carbon-rich composition (this work is a good example of the stellar history which can be deduced from observations of the envelope properties at different radii). (2) The J-type carbon stars also have high values of $13 \mathrm{C} / 12 \mathrm{C}$ ( 1:10, e.g. Lambert et al. (1986). While many carbon stars have low values of $13 \mathrm{C} / 12 \mathrm{C}(\sim \overline{1: 50-150}$, Knapp and Chang 1985, Lambert et al. 1986) due to the dredge-up of $3 \alpha$ burning products, oxygen stars have much higher values ( $1: 4-20$, references cited above) due to the production of ${ }^{3} \mathrm{C}$ during main sequence CNO burning. Again, these data suggest that J-type carbon stars may be evolving from oxygen to carbon-rich composition.

The fraction of AGB stars which are found to be carbon stars varies depending on the technique used for the observations:

\section{Sample}

Optical AGB stars

IRAS sources, $\mathrm{S}_{12} \mu_{60^{\circ}} 28 \mathrm{Jy}$

Stars with IRAS LRS spectra $(>2 \mathrm{Jy}$ at $12 \mu)$

AGB stars detected in CO lines

Planetary nebulae with wel1observed abundances
Carbon Stars as

Fraction of Total

\section{Reference}

few $\% \quad$ Feast (1987)

$13 \% \quad$ Hacking et al. (1985)

$25 \% \quad$ Knapp \& Wilcots (1987)

$25 \%$ IRAS Science Team (1986)

$\sim 50 \%$

$\sim 60 \% \quad$ Zuckerman \& Aller (1986)

Carbon grains are strongly absorbing relative to silicate grains

(Draine and Lee 1984) so it is not surprising that carbon stars are poorly represented in optical surveys. On the other hand, the CO abundance is greater in carbon than in oxygen stars and so carbon stars are more easily detected by $\mathrm{CO}$ observations. Nevertheless, the above compilation suggests that in total carbon stars may be significant fraction (close to $50 \%$ ?) of all AGB stars, contribute a significant fraction of returned mass to the interstellar medium, and form a significant fraction of the planetary nebulae.

\section{MOLECULAR ENVELOPES AROUND PLANETARY NEBULAE}

The link between AGB stars and planetary nebulae is most directly seen from the presence of the remnants of the AGB molecular envelopes around some planetaries, the best known case being that of NGC7027 (Mufson, Lyon and Marionni 1975). The expansion of the ionization front into the neutral circumstellar cloud has been measured by Masson (1986) for this nebula and by Kwok and Feldman (1981) for CRL618. Further evidence of the interaction between ionization fronts and circumstellar 
material is seen in emission from shock-excited $\mathrm{H}_{2}$ in many nebulae (e.g. Zuckerman and Gatley 1987).

In addition to planetary nebulae with circumstellar molecular clouds, there are several circumstellar envelopes surrounding stars of much earlier type than $M$, and these may be evolving towards the planetary nebula phase. Examples of such objects are IRC+10420 (whose envelope is oxygen rich) and CRL2688 (carbon rich). CO observations of other possible examples of this class (Parthasarathy and Pottash 1986) have found molecular circumstellar material in the CO line (Likkel et al. 1987). A list of planetary and proto-planetary nebulae detected in the co line is given below.

Co Observations of Planetary and Proto-Planetary Nebulae

$\begin{array}{lcccl}\text { Object } & \dot{\dot{M}} & & & \\ & \left(M_{\odot} y^{-1}\right) & \text { Type } & \text { Chemistry } & \text { Reference } \\ \text { CRL618 } & 7.7 \times 10-5 & \text { PN } & \mathrm{C} & 1 \\ \text { NGC2346 } & 1.0 \times 10-5 & \text { PN } & \text { C } & 2,3,4,5 \\ \text { Vy2-2 } & 2.4 \times 10-6 & \text { PN } & 0 & 1,6 \\ \text { IRC+10420 } & 3.0 \times 10-4 & \text { PPN } & 0 & 1,7,8 \\ \text { M1-92 } & 3.3 \times 10-5 & \text { PPN } & 0 & 6,9 \\ \text { NGC7027 } & 1.1 \times 10-4 & \text { PN } & \text { C } & 1.2 \\ \text { CRL2688 } & 1.6 \times 10-4 & \text { PPN } & \text { C } & 1 \\ \text { NGC6302 } & 5.7 \times 10-5 & \text { PN } & 0 & 10,12 \\ \text { NGC7293 } & \sim 10-5 & \text { PN } & \text { C } & 2,11 \\ \text { NGC6720 } & \sim 2 \times 10-5 & \text { PN } & 0 & 2,4 \\ \text { CPD-56 } 8032 & 1.3 \times 10-6 & \text { PN } & C & 13 \\ \text { HD161796 } & 10-5 & \text { PPN } & & 14 \\ 89 \text { Her } & 6 \times 10-7 & \text { PPN } & & 14 \\ \text { CRL2343 } & 7 \times 10-5 & \text { PPN } & & 14 \\ \text { SA0163075 } & 4 \times 10-6 & \text { PPN } & C ? & 13,14\end{array}$

References: 1. Knapp and Morris 1985, and references therein, 2. Pottasch 1980, 3. Walsh 1982, 4. Huggins and Healy 1986b, 5. Knapp 1986,6 . Seaquist and Davis 1983, 7. Bowers 1984, 8. Diamond et al. 1985, 9. Davis et al. 1979, 10. Zuckerman and Dyck 1987, 11. Huggins and Healy $1986 a, 12$. Payne, Phillips and Terzian 1987, 13. Phillips et al. 1987,14 . Likkel et al. 1987.

Here, a planetary nebula is defined as one in which ionized gas is already present, while a proto-planetary nebula is a molecular cloud surrounding an evolved star of type earlier than M (e.g. 89 Her is an F supergiant) but in which the ionization of the gas has not yet begun. There are eight planetaries currently detected in the CO lines. Of special interest is the recent $C O$ detection of the probable low-mass planetary nebula CPD $-56^{\circ} 8032$ (Phillips et al. 1987).

The objects listed in the above table have both oxygen-rich chemistry (e.g. NGC6302, Vy2-2) and carbon-rich chemistry (e.g. NGC7027, CPD-56 8032 ). Further, they have a range of progenitor masses. The total mass of NGC7027 is likely to be $>3 \mathrm{M}_{\odot}$, while 
CPD $-56^{\circ} 8032$ may be a population II object. Thus carbon stars, like $M$ stars have a range of progenitor masses, and both oxygen and carbon stars become planetary nebulae. Oxygen and carbon stars may form a parallel sequence on the $A G B$, and not all oxygen stars become carbon stars.

\section{EVOLUTION TO PLANETARY NEBIJLAE}

There is increasing evidence that, whatever the fundamental cause of mass loss on the AGB, the envelope dynamics are dominated by radiation pressure on dust grains which form in the extended stellar atmospheres. A requirement for radiation pressure driven winds is $\dot{M}_{0}<\mathrm{kL}_{*} / \mathrm{c}$, where $k$ is a number of order 1 and $L_{*}$ is the stellar luminosity. Values of $M$ can be found from $\mathrm{CO}$ observations; since both the observed $\mathrm{CO}$ ) line strength and the stellar bolometric flux are $\sim D^{-2}$, where $D$ is the distance, one can take the ratio $\beta=M_{0} c / L_{*}$ and require that $\beta<1$. In this way, the distance, the largest source of uncertainty, drops out of the problem. A compilation of mass loss rates by Knapp (1986) showed that a very large fraction of $A G B$ stars detected in molecular line emission have values of $B$ within a factor of 3 (the observational uncertainty) of $l$ while the remainder have $\beta<1$. Thus a majority of the envelopes from which molecular enission is detected are losing mass at the radiation pressure limit.

There are, however, a few exceptions with $\beta \gg 1$. All of these have central ionized regions (i.e. are young planetary nebula) and all of the nebulae in the sample have $\beta \gg 1$ except Vy2-2 and CPD-56 8032 . This observation allows us to exploit the information about the evolutionary history of the star carried by the stellar wind. The molecular line observations tell us what the mass-loss rate used to be $\sim 10^{4}$ years ago, while the bolometric flux tells us what the stellar luminosity is now. The value of $\beta$ thus tells us the minimum luminosity drop between the red giant branch and the present evolutionary status of the star. Figure 4 shows $\beta$ versus the present spectral type of the central star. We can see stars both on Schonberner's (1987) H-burning (constant luminosity) tracks as well as stars for which the luminosity drops abruptly at the onset of planetary nebula formation, and are therefore fairly massive (cf. Paczyñski 1971).

I am very grateful to Tom Phillips, Hans Olofsson, Mike Jura and Ben Zuckerman for access to their data before publication. This work is supported by NSF grant AST87-02945 to Princeton University. 


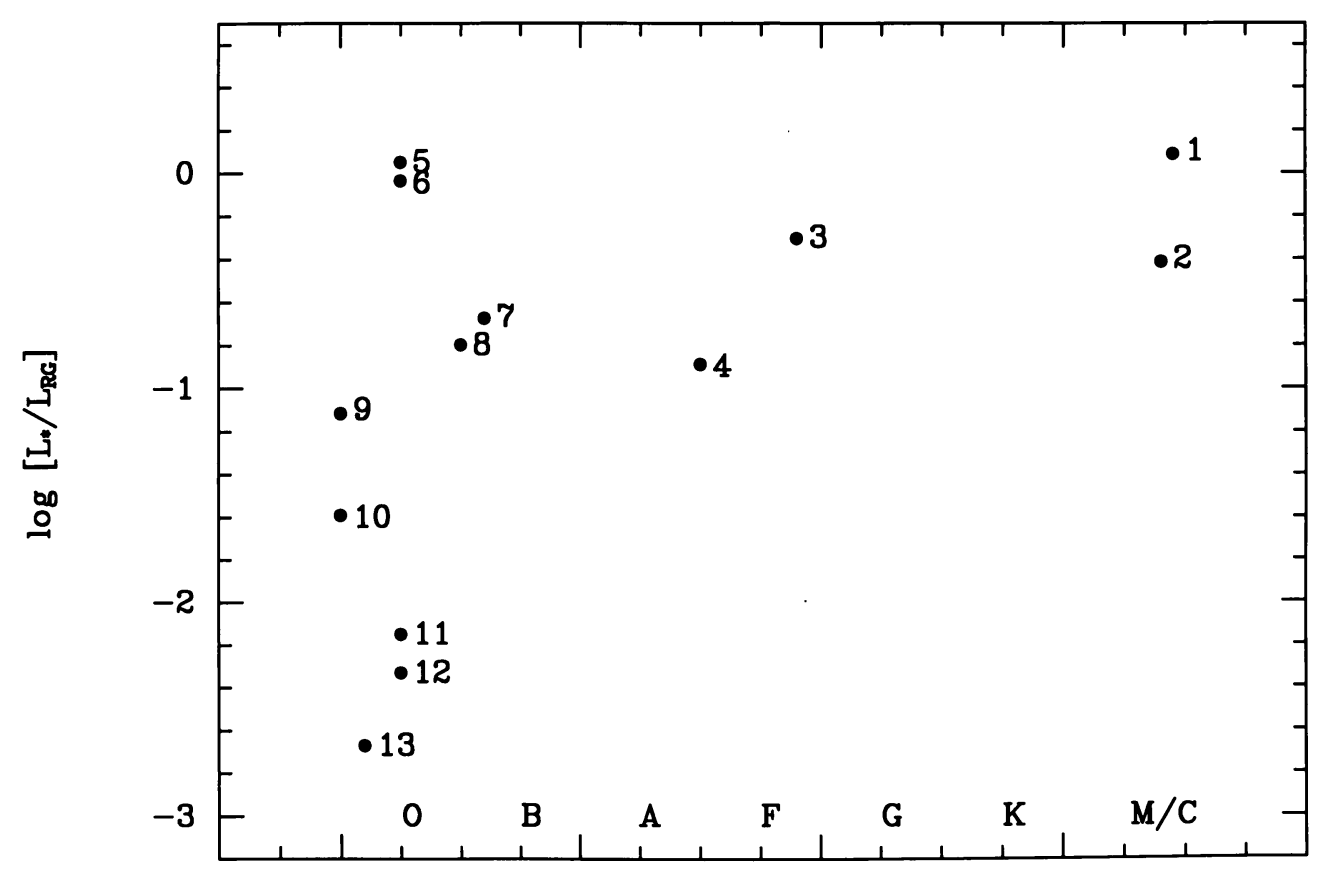

\section{Spectral Type}

Figure 4.- Luminosity decrease for PN and PPN versus approximate spectral type. The stars are (1) and (2) IRC +10011 and IRC+10216, mass losing AGB stars (3) IRC+10420

(4) CRL2688 (5) CPD-56 ${ }^{\circ} 8032$ (6) Vy2-2 (7) M1-92 (8) CRL618

(9) N7027 (10) N6302 (11) N7293 (12) N6720 (13) N2346.

\section{References}

Arquilla, R., Leahy, D.A., and Kwok, S. 1986, M.N.R.A.S. 220, 125. Bowers, P.F. 1984, Ap.J. 279, 350.

Claussen, M.J., Kleinmann, S.G., Joyce, R.R., and Jura, M. 1987, Ap.J. (Suppl) (in press).

Davis, L.E., Seaquist, E.R., and Purton, C.R. 1979, Ap.J. 230, 434.

Diamond, P.J. et al. 1985, M.N.R.A.S. 212, 1.

Draine, B.T., and Lee, H.-M. 1984, Ap.J. 285, 89.

Eder, J., Lewis, B.M., and Terzian, Y. 1987, Ap.J. (in press).

Feast, M.W., 1987, in "The Outer Galaxy", ed. F.J. Lockman and L.

Blitz, Springer-Verlag, in press.

Habing, H.J., and te Lintel Hekkert, P. 1987, in preparation. 
Hacking, P., et al. 1985, P.A.S.P. 97, 616.

Huggins, P.J., and Healy, A.P. 1986a, Ap.J. (Letters) 305, L29. $1986 \mathrm{~b}, \mathrm{M} \cdot \mathrm{N} \cdot \mathrm{R} \cdot \mathrm{A} \cdot \mathrm{S} \cdot 220$, 33p.

IRAS Point Source Catalog 1985, N.A.S.A., U.S. Government Printing office.

IRAS Science Team 1986, Astron. Astrophys. Supp1. 65, 607.

Knapp, G.R. 1986, Ap.J. 311 , 731 .

Knapp, G.R., and Chang, K.-M. 1985, Ap.J. 293, 281.

Knapp, G.R., and Morris, M. 1985, Ap.J. 292, 640.

Knapp, G.R., and Wilcots, E.P. 1987, in preparation.

Kwok, S., and Feldman, P.A. 1981, Ap.J. (Letters) 247, L67.

Lambert, D.L., Gustafsson, B., Eriksson, K., and Hinkle, K.H. 1986, Ap.J. (Supp1.) $62,373$.

Leahy, D. A., Kwok, S., and Arquilla, R.A. 1987, Ap.J. 320, 825.

Likkel, L., Omont, A., Morris, M., and Forveille, T. 1987, Astron. Astrophys. 173, L11.

Little-Marenin, I.R. 1986, Ap.J. (Letters) 307 , L15.

Masson, C.R. 1986, Ap.J. (Letters) 302, L27.

Mihalas, D., and Binney, J. 1981, Galactic Astronomy (W.H. Freeman Co.).

Mufson, S.L., Lyon, J., and Marionni, P.A. 1975, Ap.J. (Letters) 201, L85.

Neugebauer, G., et al. 1984, Ap..J. (Letters) 278, L1.

01non, F.M. et a1. 1984, Ap.J. (Letters) 278, L41.

Olofsson, H., Eriksson, K., and Gustafsson, B. 1987, Astron. Astrophys. 183 , L13.

Paczyñski, B.E. 1971, Act. Astron. 21, 271.

Payne, H.E., Phillips, J.A., and Terzian, Y. 1987, Ap.J.. (in press).

Parthasarathy, M., and Pottasch, S.R. 1986, Astron. Astrophys. 154 ,

L16.

Phillips, T.G. et al. 1987, submitted to Ap.J.

Pottasch, S.R. 1980, Astron. Astrophys. 89, 336.

Rieu, N.G., Epchtein, N., Bach, T., and Cohen, M. 1987, Astron.

Astrophys. (in press).

Seaquist, E.R., and Davis, L.E. 1983, Ap.J. 274, 659.

Schonberner, D. 1987, in "Late Stages of Steilar Evolution" ed. S. Kwok and S.R. Pottasch, D. Reidel Co.

Thronson, H.A., Latter, W.B., Black, J.H., Bally, J., and Hacking, P. 1987, Ap.J. (in press).

Walsh, J. R. 1982, M.N.R.A.S. 202, 303.

Wannier, P.G., and Sahai, R. 1986, Ap.J. 311, 335.

Willems, F., and de Jong, T. 1986, Ap.J. (Letters) 309, L39.

Willems, F., and de Jong, T. 1987, Astron. Astrophys. (in press).

Zuckerman, B., and Aller, L. 1986, Ap.J. 301, 772.

Zuckerman, B., and Dyck, H.M. 1986a, Ap.J. 304, 394.

$1986 \mathrm{~b}$, Ap.J. $311,345$.

1987 , Ap.J. (in press).

Zuckerman, B., Dyck, H.M., and Claussen M.J. 1986, Ap.J. 304, 401.

Zuckerman, B., and Gatley, I. 1987, Ap.J. (in press). 